\title{
ELEMENTARY EXCITATIONS IN ANTIFERROMAGNETIC HEISENBERG SYSTEMS
}

\author{
P.L. ISKE and W.J. CASPERS \\ Center for Theoretical Physics, University of Twente, Enschede, The Netherlands
}

Received 11 March 1987

\begin{abstract}
The structure of the (eigen)states of antiferromagnetic Heisenberg systems is discussed. These systems are shown to be equivalent to classical systems of coupled harmonic oscillators. Most attention will be paid to the first excited state. This state is supposed to be a triplet. An approximation method, which is a generalization of a method, used to describe the ground state of Heisenberg systems, will be used to describe elementary excitations. The working of the method is demonstrated by some small-system calculations.
\end{abstract}

\section{Introduction}

Antiferromagnetic Heisenberg systems (with spin $\frac{1}{2}$ ) have been studied extensively in the literature. Roughly speaking there are two fields of interest:

The first category concerns the ground-state properties, e.g. the ground-state energy, the occurrence of symmetry breaking, etc. Important results here are the calculation of the ground-state wave function for a chain, done by Bethe in $1931^{1}$ ), the proof of the theorem that states that no long-range order can exist in one- and two-dimensional systems at finite temperatures, by Mermin and Wagner ${ }^{2}$ ), while Peierls and Marshall proved that for a special class of antiferromagnetic Heisenberg systems, the ground state is a singlet ${ }^{3}$ ).

In this work we will mainly be interested in the second field, i.e.: the excited states, in particular in elementary excitations. Points of interest will be: Is there a gap in the energy spectrum between the ground state and the first excited state? Furthermore we will try to say something more about the wave function of the first excited state and we will try to make plausible that the first excited state is a triplet.

Des Cloizeaux and Pearson ${ }^{4}$ ) used a spin-wave method to describe elementary excitations (the spin of an elementary excitation is supposed to have the value $S=1$ ). From their calculations it followed that there is no gap in the spectrum of a one-dimensional spin- $\frac{1}{2}$ Heisenberg system.

0378-4371/87/\$03.50 C Elsevier Science Publishers B.V. (North-Holland Physics Publishing Division) 
In this work we will use another 'language' to describe (elementary) excitations. For that purpose, each state of the system will be characterized by the sign of the coefficients, belonging to the (Ising) configurations that together constitute this state. These signs are to be compared with the corresponding ones in the ground state, which are predicted by the so-called Marshall criterion $^{3}$ ). Many features of these states can be visualized by using the fact that each Heisenberg system corresponds to a classical system of coupled harmonic oscillators (this will be shown in section 2 ). This equivalence will be used, e.g. to justify the assumption that the first excited state is a triplet state.

In section 6, an approximation method to describe elementary excitations will be introduced. This method, which can be seen as an extension of a method to describe the ground state, introduced by Iske and Caspers ${ }^{5}$ ), is based on dimer coverings on the Heisenberg lattice. The working of the method will be demonstrated by some small-system calculations (cf. section 6). This approximation, which accuracy depends on the dimension (it is supposed to be more reliable in higher-dimensional systems), may account for the absence of a gap in the energy spectrum. This conjecture is supported by the fact that some consequences of the method are in agreement with features, predicted by an analysis of the (eigen) states of a Heisenberg system, done in the sections 2,3 and 5 .

\section{Equivalence between Heisenberg systems and systems of coupled harmonic oscillators}

In this section we will show that the quantum-mechanical Heisenberg model is equivalent to a classical model of coupled harmonic oscillators. In this equivalence the amplitudes for a basis in the space of quantum states of the Heisenberg model correspond with the classical amplitudes of a set of coupled harmonic oscillators. We consider a lattice of $2 N$ spins $\frac{1}{2}$. The coupling parameter between two spins $i$ and $j$ is $J_{i j}$. The Heisenberg hamiltonian can be written as

$$
\begin{aligned}
\mathbf{H} & =\sum_{\langle i j\rangle} J_{i j} s_{i} \cdot s_{j} \\
& =\sum_{\langle i j\rangle} J_{i j} s_{i}^{z} s_{j}^{z}+\frac{1}{2} \sum_{\langle i j\rangle} J_{i j}\left(s_{i}^{+} s_{j}^{-}+s_{i}^{-} s_{j}^{+}\right),
\end{aligned}
$$

where the summations run over all pairs of spins.

Together with the total spin $S$, one can choose the $z$-component $M$ as a good quantum number and for studying the energy spectrum one can restrict oneself to the subspace $\boldsymbol{\Omega}$ corresponding with $M=0$, in which all eigenvalues of $\mathbf{H}$ 
have a representing eigenvector. As an orthonormal basis for $\boldsymbol{\Omega}$ we choose the set of Ising configurations, i.e. states with as many spins 'up' as 'down', represented by kets like $|++-+-\cdots \cdots\rangle$. The \pm sign on the $i$ th place stands for an eigenvalue $\pm \hbar$ of $s_{i}^{z}$. The number of these states, $\mathscr{L}$, which is also the dimension of $\boldsymbol{\Omega}$, is given by

$$
\mathscr{L}=\operatorname{dim}(\boldsymbol{\Omega})=\frac{(2 N) !}{N ! N !}=\left[\begin{array}{c}
2 N \\
N
\end{array}\right] .
$$

We can now give the matrix elements of $\mathbf{H}$ in this basis. The first summation in (1) gives us the diagonal elements (taking $\hbar=1$ from now on)

$$
\begin{aligned}
h_{n n} & =\left\langle\varphi_{n}|\mathbf{H}| \varphi_{n}\right\rangle=\frac{1}{4}\left[\sum_{\langle i j\rangle_{\|}} J_{i j}-\sum_{\langle i j\rangle_{\nVdash}} J_{i j}\right] \\
& =\frac{1}{4} \sum_{\langle i j\rangle} J_{i j}-\frac{1}{2} \sum_{\langle i j\rangle_{\nVdash}} J_{i j}=k-\frac{1}{2} \sum_{\langle i j\rangle_{\nVdash}} J_{i j} .
\end{aligned}
$$

In (3), the symbol $\Sigma_{\|}$means the sum over all parallel spin pairs in the Ising configuration $\left|\varphi_{n}\right\rangle$, while $\Sigma_{\|}$stands for summation over all anti-parallel spin pairs. Notice that $k$ is, except for a factor 4 , just the sum of all coupling constants. The non-diagonal elements of $\mathbf{H}$ are given by the second summation in (1),

$$
h_{n m}=\left\langle\varphi_{n}|\mathbf{H}| \varphi_{m}\right\rangle=\frac{1}{2} J_{i j}=h_{m n},
$$

for $\left|\varphi_{n}\right\rangle$ and $\left|\varphi_{m}\right\rangle$, 'connected' by the anti-parallel spin pair $\langle i j\rangle$; i.e. $\left|\varphi_{n}\right\rangle$ can be obtained from $\left|\varphi_{m}\right\rangle$ (and vice versa) by interchanging the anti-parallel spins $i$ and $j$. If $\left|\varphi_{n}\right\rangle$ and $\left|\varphi_{m}\right\rangle$ are not connected by such a pair we get

$$
h_{n m}=h_{m n}=0
$$

From (3), (4) and (5) it follows that

$$
h_{n n}=k-\sum_{m(\neq n)} h_{n m},
$$

so $\mathbf{H}$ has the following, symmetric form:

$$
\mathbf{H}=\left[\begin{array}{cccc}
k-\sum_{i \neq 1} h_{1 i} & h_{12} & \cdots & h_{1 \mathscr{L}} \\
h_{21} & k-\sum_{1 \neq 2} h_{2 i} & \cdots & h_{2 \mathscr{L}} \\
\vdots & \vdots & & \vdots \\
h_{\mathscr{L} 1} & h_{\mathscr{L} 2} & \cdots & k-\sum_{i \neq \mathscr{L}} h_{\mathscr{L} i}
\end{array}\right]
$$


We can split up $\mathbf{H}$ into two matrices $\mathbf{K}$ and $\mathbf{H}^{\prime}$,

$$
\mathbf{H}=\mathbf{K}-\mathbf{H}^{\prime} \text {, }
$$

where $\mathbf{K}$ is $k$ times the $(\mathscr{L} \times \mathscr{L})$-unit matrix and $\mathbf{H}^{\prime}$ is the symmetric matrix:

$$
\mathbf{H}^{\prime}=\left[\begin{array}{cccc}
\sum_{i \neq 1} h_{1 i} & -h_{12} & \cdots & -h_{1 \mathscr{f}} \\
-h_{21} & \sum_{i \neq 2} h_{2 i} & \cdots & -h_{2 \mathscr{L}} \\
\vdots & \vdots & & \vdots \\
-h_{\mathscr{Z} 1} & -h_{\mathscr{W} 2} & \cdots & \sum_{i \neq \mathscr{L}} h_{\mathscr{L}}
\end{array}\right]
$$

We will further restrict ourselves to those cases where

$$
\underset{i, j}{\forall} J_{i j} \geqslant 0,
$$

so we are dealing with an antiferromagnetic Heisenberg model. Now the matrix $\mathbf{H}^{\prime}$ is of a special kind: The sum of all elements in each row and in each column is zero. Furthermore the diagonal elements are the only positive elements. Kirchhoff ${ }^{6}$ ) used matrices with these properties to describe the structure of linear resistor networks. All important features of the network R, c.g. the resultant resistance between two arbitrary nodes, can be expressed in terms of $\mathbf{H}^{\prime}(\mathbf{R})$, where $h_{i j}$ is the conductivity of the resistor coupling the nodes $i$ and $j ; h_{i j}=0$ if $i$ and $j$ are not directly connected by a resistor.

Now it will be clear that there is an equivalence between the antiferromagnetic Heisenberg model and linear networks: We can interpret the basis of Ising configurations as the $\mathscr{L}$ nodes of a network $\mathbf{R}$. The coupling parameters (4) between two Ising configurations should be interpreted as the conductivities between the corresponding nodes in $\boldsymbol{R}$. In the appendix, fig. A.1b, we have given the "configuration network" $\mathbf{R}$ corresponding to the Heisenberg model for a ring with 6 spins. There are 20 Ising configurations, listed in table A.I. Of course, in general $\mathbf{R}$ will be very complicated.

Now we consider a classical system of harmonic oscillators, all with the same mass $m$, situated on the $\mathscr{L}$ nodes of the configuration network $\mathbf{R}$. The coupling parameter between the oscillators in the nodes $i$ and $j$ is $h_{i j}$. For the displacements $x_{i}(i=1,2, \ldots, \mathscr{L})$ from the equilibrium positions we get $\mathscr{L}$ differential equations,

$$
m\left(\partial^{2} x_{i} / \partial t^{2}\right)=-\sum_{j(\neq i)} h_{i j}\left(x_{i}-x_{j}\right) .
$$


The eigenmodes are

$$
x_{i}(t)=x_{i}(0) \mathrm{e}^{\mathrm{i} \omega t}
$$

Inserting (12) into (11) gives us $\mathscr{L}$ equations,

$$
m \omega^{2} x_{i}(0)-\sum_{j \neq i)} h_{i j}\left[x_{i}(0)-x_{j}(0)\right]=0 .
$$

The $\mathscr{L}$ solutions $m \omega_{i}^{2}$ (some of them may be degenerate) of the set equations (13) are the eigenvalues of the matrix $\mathbf{H}^{\prime}$, given in (9). The conclusion is therefore that both problems, solving the eigenvalue problem for the Heisenberg hamiltonian as well as finding the eigenfrequencies and eigenmodes of a (corresponding) classical system of coupled harmonic oscillators, can be reduced to solving the eigenvalue problem for the same Kirchhoff matrix, so these problems can be mapped onto each other. We will use this equivalence to visualize some of the features of the eigenstates of the Heisenberg system in the next sections.

\section{3. "Land/water" structure of the (eigen)states of an antiferromagnetic Heisen- berg system}

Throughout this work we will only consider those Heisenberg lattices that can be divided into two sublattices $\alpha$ and $\beta$, each of them containing $N$ spins. Only interactions between two spins on different sublattices are possible and all interactions $\neq 0$ have the uniform value $J(J>0)$. An important theorem about the structure of the ground state of a system of this type has been proven by Marshall $^{3}$ ). This theorem predicts the relative sign of the coefficients of the Ising configurations contributing to the ground state.

We write an eigenvector of the hamiltonian (1) as

$$
\left|\Psi_{m}\right\rangle=\sum_{i=1}^{\mathscr{L}} c_{i}\left|\varphi_{i}\right\rangle,
$$

where the sum runs over the Ising configurations of $\boldsymbol{\Omega}$. Following Marshall, we introduce two quantities for each Ising configuration $\left|\varphi_{i}\right\rangle$ :

$-q_{i}$ equals the number of antiparallel nearest neighbour pairs (=pairs of spins with interaction $J$ );

$-p_{i}$ is the number of + spins on the $\alpha$-sublattice.

The total number of nearest neighbour pairs equals $Z$. The eigenvectors of Kirchhoff matrix may always chosen to be real (while it is a real symmetric 
matrix), so $c_{i}=c_{i}^{*}$ in (14). Furthermore we suppose $\left|\Psi_{m}^{\prime}\right\rangle$ to be normalized. The $q_{i}$ Ising configurations, connected to $\left|\varphi_{i}\right\rangle$ by interaction of an antiparallel pair (in the way as discussed in section 2), are written as $\left|\varphi_{i}^{k}\right\rangle$ and have coefficients $c_{i}^{k}\left(k=1,2, \ldots, q_{i}\right)$. Using (3) and (4) we can derive the following expression for the expectation value of the energy for a normalized linear combination of Ising configurations $\left|\Psi_{m}\right\rangle$ :

$$
E_{m}=\left\langle\Psi_{m}|\mathbf{H}| \Psi_{m}\right\rangle=\frac{1}{4} Z J+\frac{1}{2} J \sum_{i} \sum_{k=1}^{q_{i}}\left(c_{i} c_{i}^{k}-c_{i}^{2}\right) .
$$

The ground state is obtained if we choose the set $\left\{c_{i}\right\}$ so that $E_{m}$ takes a minimum value. Marshall's theorem is that this set must have the form

$$
c_{i}=(-1)^{p_{i}} a_{i}, \quad a_{i}>0
$$

Only in that case each term $c_{i} c_{i}^{k}$ in (15) is negative (while $p_{i}^{k}=p_{i} \pm 1$, $k=1,2, \ldots, q_{i}$ ), so (16) is a necessary condition for $E_{m}$ to take its minimum value for given values of $\left|c_{i}\right|$. Because all Ising configurations have a non-zero coefficient, the ground state is non-degenerate. If we consider a Heisenberg system with its corresponding configuration network $\mathbf{R}$, as defined in section 2 , Marshall's theorem shows us that in the ground state the coefficients of two connected Ising configurations must have opposite signs. We are free to choose the sign of one of the coefficients (for instance we can give the "first" Néel configuration, $|+-+-\cdots\rangle$, a positive sign), but after doing that, all other signs are fixed. On grounds of orthogonality of the eigenstates the Marshall condition (16) cannot hold for any other eigenstate of $\mathbf{H}$, so in each excited state there are Ising configurations with a "correct" sign (we will call these configurations "land configurations" throughout this work) while others ("water configurations") have a "wrong" sign compared with the corresponding one in the ground state. There is still an arbitrariness in what is "correct" and what is "wrong", but when we have chosen the sign of one of the Ising configurations it is clear which configurations are "land" and which are "water". Of course it is possible that there are Ising configurations with coefficients zero. It is obvious that we can define this land/water structure for each real state $|\Psi r\rangle$ in $\mathbf{\Omega}$ (which need not be a eigenstate of $\mathbf{H}$ ). If it happens that there are Ising configurations not contributing to $|\Psi\rangle$, so with a coefficient zero, we define the land/water structure on the reduced network $\mathbf{R}|\Psi\rangle$, which can be obtained from $\mathbf{R}$ by omitting the nodes, corresponding with these zero-coefficient configurations and the connecting bonds. It is clear that if there are no such configurations we get $\mathbf{R}|\Psi\rangle=\mathbf{R}$. Now the network $\mathbf{R}|\Psi\rangle$ falls apart into two types of connected subsets of Ising configurations: 
- "Land areas": A land area $L$ is a maximum subset of connected land configurations.

- "Water areas": A water area $W$ is a maximum subset of connected water configurations.

Boundary configurations are Ising configurations connected to one or more configurations in $\mathbf{R}|\Psi\rangle$ not belonging to the same area. These last configurations are boundary configurations too! If two (boundary) configurations are connected and they belong to different areas, one of them must be land and the other must be water. This fact explains the used terminology.

For an example of a land/water structure (corresponding to the first excited stated of a closed chain of 6 spins, cf. section 5), we refer to appendix A, fig. A.1c.

Marshall $^{3}$ ) proved the ground state to be a singlet state. Lieb and Mattis ${ }^{7}$ ) generalized this statement. Their result can be formulated as follows: The ground state of an antiferromagnetic Heisenberg system of the type, defined in the first paragraph of this section, in the subspace of states with the $z$ component of the total spin having the value $M$ is a state with $S=M$. For each value of $M$ we can give the matrix elements of $\mathbf{H}$ in the corresponding basis of "Ising configurations" (states like $|++--+\cdots\rangle$ with $S^{z}=M$ ). The structure of $\boldsymbol{H}$ in these bases is the same as in the case $M=0$, so again the ground state, which has the lowest possible total spin $S=M$, is "Marshall", i.e., two configurations connected by a matrix element of $\mathbf{H}$ have coefficients with opposite sign. This state is non-degenerate too, while all configurations have a non-zero coefficient. Of course we can introduce a configuration network corresponding to each $M$-subspace. The land/water structure of the ground state in each $M$-subspace is made up of just one area.

Now we discuss the structure of the highest excited state of the antiferromagnetic Heisenberg system. The lowest eigenvalue of the Kirchhoff matrix $\mathbf{H}^{\prime}$ (cf. (9)) is zero, so the maximum eigenvalue of the hamiltonian (7) is (cf. (3))

$$
E_{\max }=k=\frac{1}{4} \sum_{\langle i j\rangle} J_{i j} .
$$

The corresponding, normalized eigenvector is

$$
\left|\Psi_{\max }\right\rangle=(1 / \sqrt{N}) \sum_{n}\left|\varphi_{n}\right\rangle
$$

Because all Ising configurations in the linear combination (18) have the same coefficient, there does not exist a single pair of Ising configurations obeying Marshall's condition, i.e. all products $c_{i} c_{i}^{k}$ in (15) are positive, so in the land/water structure of $\left|\Psi_{\max }\right\rangle$ each Ising configuration constitutes an isolated 
area of its own! This last statement is true in each $M$-subspace $\left(\left|\Psi_{\max }\right\rangle\right.$ has the total maximum spin $S=N$, so has a representative in each subspace), because in each $M$-subspace the hamiltonian can be decomposed as in (8) with $\mathbf{H}^{\prime}$ being a Kirchhoff matrix.

Now the connection to the model of coupled harmonic oscillators, as discussed in section 2 , becomes more clear. We consider in the harmonicoscillator system the standing wave, corresponding to a state $|\Psi\rangle$ of the Heisenberg system. The amplitudes of the oscillators correspond with the amplitudes of the corresponding Ising configurations contributing to $|\Psi\rangle$, so if two connected Ising configurations $\left|\varphi_{n}\right\rangle$ and $\left|\varphi_{m}\right\rangle$ are "Marshall", i.e. $c_{n} c_{m}<$ 0 , then there is a node between the corresponding oscillators. Roughly speaking one can say that the frequency of a harmonic-oscillator system is increasing with an increasing number of nodes, so we are not surprised that the structure of the ground state (that corresponds with the maximum eigenfrequency) contains only one area (i.e. the maximum number of nodes) and that of the highest excited state the maximum number of areas (no nodes).

\section{Time reversal (TR) and a decomposition of $\mathbf{\Omega}$}

All eigenvalues of the hamiltonian have a representing eigenvector in the $M=0$ subspace $\boldsymbol{\Omega}$. These eigenstates together constitute an orthonormal basis for $\mathbf{\Omega}$. We can divide the eigenstates into two classes: those with an even total spin $S$ form a basis for the subspace $\boldsymbol{\Omega}^{\text {even }}$ of $\boldsymbol{\Omega}$; those with an odd value for $S$ are a basis for the subspace $\boldsymbol{\Omega}^{\text {odd }}$, the orthogonal complement of $\boldsymbol{\Omega}^{\text {even }}$ in $\boldsymbol{\Omega}$. So we write

$$
\boldsymbol{\Omega}=\boldsymbol{\Omega}^{\text {even }} \oplus \boldsymbol{\Omega}^{\text {odd }}
$$

We will show that $\boldsymbol{\Omega}^{\text {even }}$ and $\boldsymbol{\Omega}^{\text {odd }}$ always have the same dimension, so the number of $M=0$ eigenstates with even total spin $S$ always equals the number of states with odd $S$.

Proof. The total space of states can be divided into subspaces $\boldsymbol{\Omega}^{M}$, corresponding to a given value $M$ of the $z$-component of the total spin $\left(\boldsymbol{\Omega}=\boldsymbol{\Omega}^{\mathbf{0}}\right)$. The dimension of $\boldsymbol{\Omega}^{\boldsymbol{M}}$ equals:

$$
\operatorname{dim}\left(\boldsymbol{\Omega}^{M}\right)=\left[\begin{array}{c}
2 N \\
N-M
\end{array}\right]
$$

For all $|M| \leqslant S$, each state with total spin $S$, which is $(2 S+1)$-fold degenerate, 
has one representative in $\boldsymbol{\Omega}^{M}$, so the number $\eta_{S}$ of states with a given spin $S$ equals

$$
\eta_{S}=\operatorname{dim}\left(\mathbf{\Omega}^{S}\right)-\operatorname{dim}\left(\boldsymbol{\Omega}^{S+1}\right)=\left[\begin{array}{c}
2 N \\
N-S
\end{array}\right]-\left[\begin{array}{c}
2 N \\
N-S-1
\end{array}\right]
$$

With the use of (21) and the identity

$$
\left[\begin{array}{c}
m \\
k
\end{array}\right]=\left[\begin{array}{c}
m-1 \\
k
\end{array}\right]+\left[\begin{array}{c}
m-1 \\
k-1
\end{array}\right]
$$

one can easily check that for odd $N$ the number of states with $S=$ even is given by

$$
\begin{aligned}
\operatorname{dim}\left(\boldsymbol{\Omega}^{\text {even }}\right) & =\eta_{0}+\eta_{2}+\eta_{4}+\cdots+\eta_{N-1} \\
& =\left[\begin{array}{c}
2 N-1 \\
N
\end{array}\right]=\frac{1}{2}\left[\begin{array}{c}
2 N \\
N
\end{array}\right]=\frac{1}{2} \operatorname{dim}(\boldsymbol{\Omega})
\end{aligned}
$$

For even $N$ we can prove in the same way that $\operatorname{dim}\left(\boldsymbol{\Omega}^{\text {odd }}\right)=\frac{1}{2} \operatorname{dim}(\boldsymbol{\Omega})$.

We will now prove a theorem that tells us something about the land/water structure of states in $\mathbf{\Omega}^{\text {odd }}$. To be able to prove this theorem we first give the proof of a lemma that tells us about the behaviour of an eigenstate of the total angular momentum under the operation of time reversal (TR). The effect of TR is that all angular momenta $J$ must be replaced by $-J$. In our language, this means that to get the time reversed of a state $|\Psi\rangle$ as given in (14), we simply must interchange the + and - signs in the contributing Ising configurations, at least in cases where all $c_{i}$ are real (and because we are working with Kirchhoff matrices this may be chosen to be so). Note that under TR the operator $\mathbf{S}^{2}$ remains unchanged, so the value of $S$ is not affected by TR. For a detailed account of TR we refer to Edmonds ${ }^{8}$ ). While the operators $\mathbf{S}^{2}, \mathbf{H}$ and $\mathbf{S}^{z}$ commute, we can denote the eigenvectors of $\mathbf{H}$ by $|S M \alpha\rangle$, with $M=$ $-S, \ldots,-1,0,1, \ldots, S$. We shall drop the degeneration index $\alpha$. We are dealing with a system of $2 N$ spins (so $S$ is an integer). The TR operator of this system is $\mathbf{P}^{2 N}$.

Lemma. There exists a basis of eigenvectors $|S M\rangle$, such that the working of $\mathbf{P}^{2 N}$ is given by

$$
\mathbf{P}^{2 N}|S M\rangle=(-1)^{N-S+M}|S-M\rangle
$$


Proof. From table I it may be clear that the lemma is true for $N=1$ :

TABLE I

Time reversal in a system of two spins $\frac{1}{2}$.

\begin{tabular}{rrlll}
\hline$S$ & \multicolumn{1}{c}{$M$} & $|S M\rangle$ & $\mathbf{P}^{2 N}|S M\rangle$ & $(-1)^{1-S+M}|S-M\rangle$ \\
\hline 0 & 0 & $(1 / \sqrt{2})(|+-\rangle-|-+\rangle)$ & $(1 / \sqrt{2})(|-+\rangle-1+-\rangle)$ & $-(1 / \sqrt{2})(|+-\rangle-|-+\rangle)$ \\
1 & -1 & $|--\rangle$ & $|++\rangle$ & $|+\rangle$ \\
1 & 0 & $(1 / \sqrt{2})(|+-\rangle+|-+\rangle)$ & $(1 / \sqrt{2})(|-+\rangle+|+-\rangle)$ & $(1 / \sqrt{2})(|+-\rangle+|-+\rangle)$ \\
1 & 1 & $-|++\rangle$ & $-|--\rangle$ & $-|-\rangle$ \\
\hline
\end{tabular}

Suppose we have proven the lemma for systems with $2,4, \ldots, 2 N$ spins. We now want to prove it for systems with $2 N+2$ spins. To do so we use the Clebsch-Gordan algebra to couple the angular momenta of system 1 (containing $2 N$ spins) with total spin $S_{1}$ and system 2 (2 spins) with spin $S_{2}$ to constitute an eigenstate $\left|\Psi\left(S_{1} S_{2} S M\right)\right\rangle$ of the $2 N+2$ system, with total spin $S$ and $z$-component $M$ (cf. ref. 8). This state can be written as a sum over direct products of eigenstates of the systems 1 and 2 ,

$$
\left|\Psi\left(S_{1} S_{2} S M\right)\right\rangle=\sum_{M_{1}, M_{2}}\left\langle S_{1} M_{1} S_{2} M_{2} \mid S_{1} S_{2} S M\right\rangle\left|S_{1} M_{1}\right\rangle \otimes\left|S_{2} M_{2}\right\rangle,
$$

where $M=M_{1}+M_{2}$. Under the condition that $\left|\Psi\left(S_{1} S_{2} S M\right)\right\rangle$ is real and normalized, it is uniquely defined by the right member of (25), apart from a \pm sign, but the choice of phase made here makes it possible to obey (24) for all $S$ and $M$. The Clebsch-Gordan coefficients $\left\langle S_{1} M_{1} S_{2} M_{2} \mid S_{1} S_{2} S M\right\rangle$ obey

$$
\left\langle S_{1} M_{1} S_{2} M_{2} \mid S_{1} S_{2} S M\right\rangle=(-1)^{S_{1}+S_{2}-S}\left\langle S_{1}-M_{1} S_{2}-M_{2} \mid S_{1} S_{2} S-M\right\rangle .
$$

Because the lemma is true for a system of $2 N$ spins (and for 2 spins) we may write

$$
\begin{gathered}
\mathbf{P}^{2 N+2}\left|S_{1} M_{1}\right\rangle \otimes\left|S_{2} M_{2}\right\rangle=\mathbf{P}^{2 N}\left|S_{1} M_{1}\right\rangle \otimes \mathbf{P}^{2}\left|S_{2} M_{2}\right\rangle \\
=(-1)^{(N+1)-S_{1}-S_{2}+M}\left|S_{1}-M_{1}\right\rangle \otimes\left|S_{2}-M_{2}\right\rangle .
\end{gathered}
$$

Using (26) and (27) to get the time reversed of $\left|\Psi\left(S_{1} S_{2} S M\right)\right\rangle$ we write 


$$
\begin{aligned}
\mathbf{P}^{2(N+1)}\left|\Psi\left(S_{1} S_{2} S M\right)\right\rangle & \\
= & \sum_{M_{1}, M_{2}}\left\langle S_{1} M_{1} S_{2} M_{2} \mid S_{1} S_{2} S M\right\rangle(-1)^{(N+1)-S_{1}-S_{2}+M}\left|S_{1}-M_{1}\right\rangle \\
& \otimes\left|S_{2}-M_{2}\right\rangle \\
= & \sum_{M_{1}, M_{2}}\left\langle S_{1}-M_{1} S_{2}-M_{2} \mid S_{1} S_{2} S-M\right\rangle(-1)^{S_{1}+S_{2}-S+(N+1)-S_{1}-S_{2}+M} \\
& \times\left|S_{1}-M_{1}\right\rangle \otimes\left|S_{2}-M_{2}\right\rangle \\
= & \sum_{\underline{M}_{1}, \underline{M}_{2}}\left\langle S_{1} \underline{M}_{1} S_{2} \underline{M}_{2} \mid S_{1} S_{2} S-M\right\rangle(-1)^{(N+1)-S+M}\left|S_{1} \underline{M}_{1}\right\rangle \otimes\left|S_{2} \underline{M}_{2}\right\rangle \\
= & (-1)^{(N+1)-S+M}\left|\Psi\left(S_{1} S_{2} S-M\right)\right\rangle,
\end{aligned}
$$

where we used $\underline{M}_{i}=-M_{i}(i=1,2)$. This completes the proof of the lemma.

Now we restrict ourselves again to the states in $\mathbf{\Omega}$, so $M=0$. From the lemma it follows that these states are eigenstates of the operator $\mathbf{P}^{2 N}$ too, with an eigenvalue $P_{S}^{2 N}$,

$$
\mathbf{P}^{2 N}|S 0\rangle=P_{S}^{2 N}|S 0\rangle=(-1)^{N-S}|S 0\rangle,
$$

so this eigenvalue $P_{S}^{2 N}$ equals

$$
P_{S}^{2 N}=(-1)^{S} P_{0}^{2 N}, \quad P_{0}^{2 N}=(-1)^{N} .
$$

Because the ground state of an antiferromagnetic Heisenberg system is a singlet, $P_{0}^{2 N}$ is the eigenvalue of $\mathbf{P}^{2 N}$ corresponding to this state.

Now we come to the proof of theorem $I$.

Theorem I. In the land/water structure of a state $|\Psi\rangle \in \mathbf{\Omega}^{\text {odd }}$ the number of land areas equals the number of water areas.

From (30) it is clear that all states with even total spin $S$ have the same TR symmetry as the ground state, while the states with an odd $S$ have an opposite TR symmetry. So, if an Ising configuration has a coefficient which has the Marshall sign, for odd $S$ the time-reversed Ising configuration has a coefficient with a sign that is the opposite of the one, given by Marshall's criterion. This implies that the time-reversed of a land (water) Ising configuration in the land/water structure of a state in $\boldsymbol{\Omega}^{\text {odd }}$ must be a water (land) configuration. So, to each land area there belongs its time-reversed water area and therefore the number of land areas equals the number of water areas. 
We are now able to discuss the structure of the ground states in $\boldsymbol{\Omega}^{\text {even }}$ and $\boldsymbol{\Omega}^{\text {odd }}$. $-\boldsymbol{\Omega}^{\text {even }}$ : The exact ground state of the system is an element of $\boldsymbol{\Omega}^{\text {even }}$, while it is a singlet, so it must be the ground state in $\boldsymbol{\Omega}^{\text {even }}$ too.

$-\boldsymbol{\Omega}^{\text {odd }}$ : For the ground state in $\boldsymbol{\Omega}^{\text {vdd }},\left|\Psi_{0}\right\rangle^{\text {odd }}$, we shall prove:

Theorem II. The land/water structure of $\left|\Psi_{0}\right\rangle^{\text {odd }}$ is made up of one land area and its time-reversed water area.

Note. From the Lieb and Mattis theorem ${ }^{7}$ ) we conclude that $\left|\Psi_{0}\right\rangle^{\text {odd }}$ is a triplet state!

Proof. Theorem I teaches us that in the land/water structure of states in $\mathbf{\Omega}^{\text {odd }}$ the number of land areas equals the number of water areas. Suppose the structure of $\left|\Psi_{0}\right\rangle^{\text {odd }}$ is such that there is more than one land area. We will show that this assumption leads to a contradiction, so the structure of $\left|\Psi_{0}\right\rangle^{\text {odd }}$, written as

$$
\left|\Psi_{0}\right\rangle^{\mathrm{odd}}=\sum_{i} c_{i}\left|\varphi_{i}\right\rangle
$$

consists of just one land area and one water area. First we notice that if there are more than two areas there cannot be any "isolated" areas, i.e. areas without boundary configurations, while in that case the ground state would be degenerate and this is not the case as we discussed in section 3, so each land area is connected to at least one water configuration and vice versa. We will now construct a state, $\left|\Phi_{0}\right\rangle^{\text {odd }}$, having a lower energy than $\left|\Psi_{0}\right\rangle^{\text {odd }}$, so the latter cannot be the ground state and hence the ground state in $\boldsymbol{\Omega}^{\text {odd }}$ should contain two areas. In the structure of $\left|\Psi_{0}\right\rangle^{\text {odd }}$ we consider a land area $L$ and its time-reversed water area $W$. We construct $\left|\Phi_{0}\right\rangle^{\text {odd }}$ by negating the coefficients corresponding to the Ising configurations in $L$ (that now becomes water) and $W$ (land). First we notice that $\left|\Phi_{0}\right\rangle^{\text {odd }} \in \boldsymbol{\Omega}^{\text {odd }}$, while we did not change the TR symmetry. In the structure of $\left|\Psi_{0}\right\rangle^{\text {odd }} L$ is only connected to water areas. Because $L$ is changed into water in $\left|\Phi_{0}\right\rangle^{\text {odd }}$, it is "melted" together with these water areas, so the number of water areas (as well as land areas) is reduced by this operation. We shall now prove that indeed the energy of $\left|\Phi_{0}\right\rangle^{\text {add }}$ is lower than the energy of $\left|\Psi_{0}\right\rangle^{\text {odd }}$, by using eq. (15) for the expectation value for the energy of a state. In (15) the double summation runs over all the connected pairs of Ising configurations. These pairs can be divided into two classes:

- I: A pair of connected Ising configurations belongs to class I, if and only if one of these configurations belongs either to $L$ or $W$. This Ising configuration is a boundary configuration of $L(W)$. If $\left|\Psi_{0}\right\rangle^{\text {odd }}$ consists of just one land area and one water area class I is empty. 
- II: All other pairs of Ising configurations belong to class II.

Now the energy (15) of $\left|\Psi_{0}\right\rangle^{\text {odd }}$ can be rewritten as

$$
E_{0}^{\mathrm{odd}}=\frac{1}{4} Z J+\frac{1}{2} J \sum_{\mathrm{I}} c_{i} c_{j}+\frac{1}{2} J \sum_{\mathrm{II}} c_{i} c_{j}-\frac{1}{2} J \sum_{i} q_{i} c_{i}^{2}
$$

All terms in the first summation in (32) are positive. However, if we change the sign of the coefficients corresponding to the Ising configurations belonging to $L$ and $W$, all these terms become negative, while all other terms in (32) are unaffected, while only in the first summation always one of the coefficients is negated. Consequently the energy of $\left|\Phi_{0}\right\rangle^{\text {odd }}$ is lower than the energy of $\left|\Psi_{0}\right\rangle^{\text {odd }}$, so the ground state in $\boldsymbol{\Omega}^{\text {odd }}$ consists of just one land area and its time-reversed water area.

We believe that this state $\left|\Psi_{0}\right\rangle^{\text {odd }}$ is also the first excited state of the Heisenberg system. (By using the Lieb and Mattis theorem we conclude that the only alternative is that the first excited state is a singlet again, with the same TR symmetry as the ground state.) Keeping the equivalence with systems of coupled harmonic oscillators in mind this seems reasonable to us, while we expect that in these systems the second-highest excited mode is one with nodes between all oscillators, except for a "frontier in the middle" (dividing the system into two symmetric parts) consisting of pairs of oscillators which are oscillating with the same phase. The assumption that the first excited state is a triplet will be our starting point for giving a possible description for elementary excitations in antiferromagnetic Heisenberg systems, as we shall do in the next section.

\section{Elementary excitations}

The aim of this section is mainly to describe elementary excitations. In particular we shall study the first excited state of an antiferromagnetic Heisenberg system. First we shall discuss some general features of excited states, illustrated by exact calculations for some small systems. For systems with a small number of spins it is possible to calculate the eigenvalues and eigenvectors of the matrix $\mathbf{H}$ (cf. (7)). Throughout this work we will give the coupling constant the uniform value $J=1$.

$N=1$ : The situation for a system of two spins is almost trivial. The ground state is a singlet, the first (and only) excited state is a triplet: 


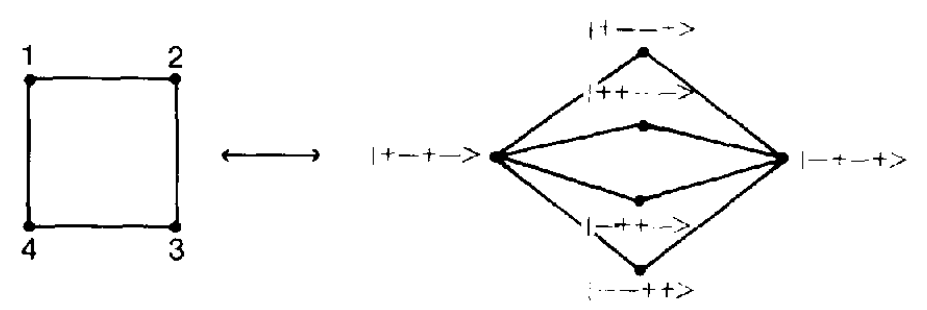

Fig. $1.2 \times 2$ square with the corresponding configuration network.

$$
\begin{aligned}
& \left|\Psi_{0}\right\rangle=(1 / \sqrt{2})(|+-\rangle-|-+\rangle), \quad E_{0}=-0.75 \\
& \left|\Psi_{1}\right\rangle=(1 / \sqrt{2})(|+-\rangle+|-+\rangle), \quad E_{1}=+0.25
\end{aligned}
$$

$N=2$ : Now we consider the $2 \times 2$ square. The corresponding configuration network is given in fig. 1.

In table II we have given the land/water structure of the eigenstates of this system. In the last column we have given the total number of areas $(\lambda)$. In this case we are able to predict the exact form of the first excited state by using theorem II. There must be one land area (including Néel $1,|+-+-\rangle$ ) and onc water area (including Néel $2,|-+-+\rangle)$. On grounds of symmetry we expect the four other Ising configurations (in the middle of fig. 1) to be absent (i.e. to have an amplitude zero). So we get

$$
\left|\Psi_{1}\right\rangle=(1 / \sqrt{2})(|+-+-\rangle-|-+-+\rangle) .
$$

$N=3$ : We also performed calculations for the three systems with 6 spins, drawn in fig. 2. By deleting the three diagonal bonds, the spherical lattice reduces to the 6-ring, whereas the latter reduces to the chain by deleting one more bond. Deleting bonds in the real Heisenberg lattice implies a reduction of the number of connections in the corresponding configuration network.

TABl.E II

The land/water structure of the states of the $2 \times 2$ square.

\begin{tabular}{lllllllllll}
\hline & $|+-+-\rangle$ & $|+--+\rangle$ & $|++--\rangle$ & $|-+++\rangle$ & $|-++\cdots\rangle$ & $|-+-+\rangle$ & $E_{i}$ & $S_{i}$ & $\lambda_{i}$ \\
\hline$\left|\Psi_{0}\right\rangle$ & $L$ & $L$ & $L$ & $L$ & $L$ & $L$ & -2 & 0 & 1 \\
$\left|\Psi_{1}\right\rangle$ & $L$ & - & - & - & - & $W$ & -1 & 1 & 2 \\
$\left|\Psi_{2}\right\rangle$ & - & - & $L$ & $W$ & - & - & 0 & 1 & 2 \\
$\left|\Psi_{3}\right\rangle$ & - & $L$ & - & - & $W$ & - & 0 & 1 & 2 \\
$\left|\Psi_{4}\right\rangle$ & - & $L$ & $W$ & $W$ & $L$ & - & 0 & 0 & 4 \\
$\left|\Psi_{5}\right\rangle$ & $L$ & $W$ & $W$ & $W$ & $W$ & $L$ & 1 & 2 & 6 \\
\hline
\end{tabular}




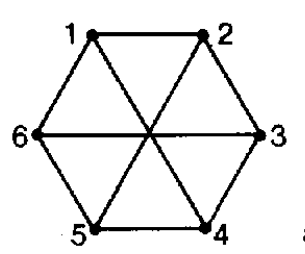

a)
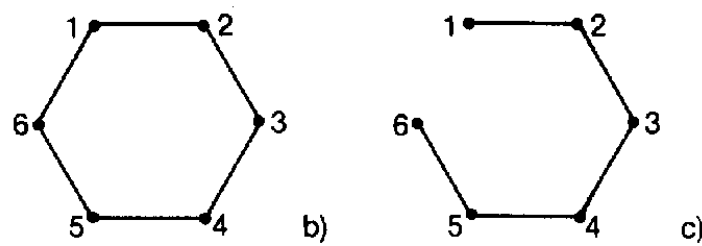

Fig. 2. Heisenberg models with 6 spins: a) spherical model, b) ring, c) chain.

\section{Features of the first excited state}

In the appendix, table A.II, we have given the land/water structure of the first excited state, corresponding to the lattices drawn in fig. 2a-c. From table A.II we conclude that the position of the node is affected by deleting connections in the configuration network. These examples illustrate the following general conclusions:

- The total spin of the first excited state seems to have always the value $S=1$.

- The first excited state of an antiferromagnetic spin- $\frac{1}{2}$ Heisenberg system has a land/water structure with one land area, consisting of Ising configurations in the "neighbourhood" of Néel configuration $1(|+-+-\rangle)$ and its timereversed water area, including Néel 2. The Néel configurations and the configurations close to them, have a large amplitude, just as in the ground state, while configurations in the "middle" of the network have a small amplitude.

$N=4$ : Calculations, performed for 8-spins systems (spherical lattice, the $2 \times 2 \times 2$-cube, the ring and the chain), also gave us an indication that these conclusions may be true. The total spin, for instance, always has the value $S=1$.

For the spherical case it is easy to predict which Ising configurations belong to which area in the first excited state. All configurations with the same $p_{i}$ (cf. section 3), i.e. with the same sublattice magnetization, are equivalent. Because there only exist connections between two configurations $\left|\varphi_{i}\right\rangle$ and $\left|\varphi_{j}\right\rangle$ for $p_{i}=p_{j} \pm 1$, we conclude that the land area consists of all configurations with $p_{i}>\frac{1}{2} N$, whereas for $p_{i}<\frac{1}{2} N$, the configuration belongs to the water area. In case of even $N$, the configurations $\left|\varphi_{i}\right\rangle$ with $p_{i}=\frac{1}{2} N$ have zero amplitudes. In general the situation is more complicated than in the spherical case, but we expect the majority of the configurations with $p_{i}>\frac{1}{2} N$ to belong to the land area, while the node is situated at $p_{i} \approx \frac{1}{2} N$. In fig. 3 we have given a schematic picture of the first excited state. In fig. 3 the dashed line symbolizes the ground state, while the first excited state is represented by the drawn line. The Ising 


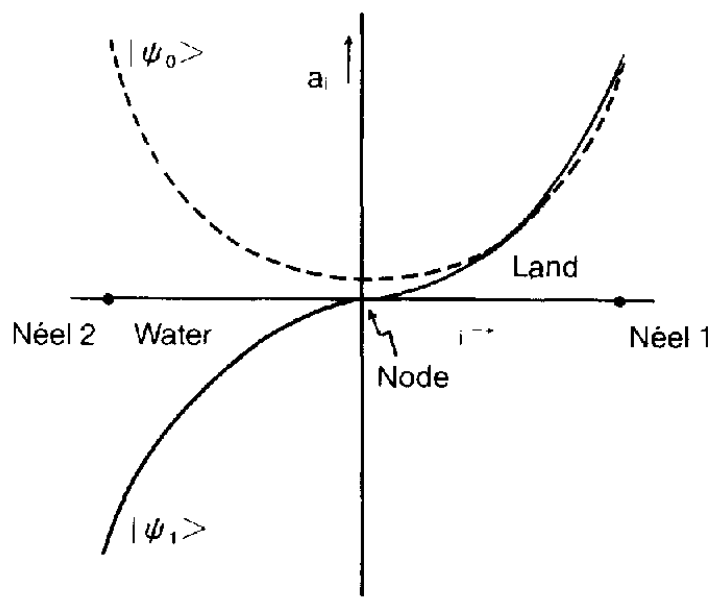

Fig. 3. Ground state and first excited state of an antiferromagnetic spin- $\frac{1}{2}$ system.

configurations are positioned along the horizontal axis; a configuration at the right is supposed to have a large $p_{i}$. We define the amplitude $a_{i}$ of an Ising configuration by generalizing (16),

$$
a_{i}=(-1)^{p_{i}} c_{i}
$$

A positive (negative) sign for the amplitude $a_{i}$ means that the coefficient of the corresponding Ising configuration has the same (opposite) sign as the corresponding coefficient in the ground state (which always has the Marshall sign, i.e. $a_{i}>0$ ).

In this picture, far from the middle, the first excited state looks like the ground state, so the energy difference between these two states is caused by the node only. Therefore no gap will be seen in the energy spectrum when the amplitudes in the middle of the network are small enough, as well in the ground state as in the first excited state. There are many indications that this is the case for large spin- $\frac{1}{2}$ systems, especially for dimensions $d \geqslant 2$ (cf. Iske and Caspers ${ }^{5}$ ).

\section{A possible construction for elementary excitations}

In a previous article we have given an approximation method for the ground state of an antiferromagnetic Heisenberg system. The method enabled us to give a possible mechanism for symmetry breaking in two- and higher dimensional systems ${ }^{5}$ ). We supposed the ground state to be a linear combination of 
singlet states. In these singlet states the spins are ordered in $(N)$ singlet pairs. In each pair there is one spin situated on the $\alpha$ sublattice and one on the $\beta$ sublattice. On the lattice we can represent such a singlet pair by a dimer, so there is a 1-1 correspondence between these singlet states ("singlet coverings") and the dimer coverings of the lattice. A natural way to construct elementary excitations is by replacing one singlet pair by a triplet pair in each singlet covering, which in principle can be done in $N$ ways, while there are $N$ dimers. Consequently the excited state (as a linear combination of "triplet coverings") is a triplet state. This recipe may be illustrated by figs. 4a,b. In fig. $4 \mathrm{a}$ a singlet covering is given, while in fig. $4 \mathrm{~b}$ one of the two corresponding triplet coverings is drawn.

According to ref. 5 we can write each singlet covering as a linear combination of Ising configurations that can be "covered" by the corresponding dimer covering, i.e. each dimer must contain one + and one - spin of the Ising configuration. According to (33a) the interchange of the + and - in a singlet pair leaves us with a minus sign, so within one covering we are automatically in agreement with Marshall's criterion. As an example we can write the singlet covering $|0\rangle$ (see fig. $4 a$ ) as

$$
|0\rangle=c\{|+-+-\rangle-|+--+\rangle-|-++-\rangle+|-+-+\rangle\} .
$$

According to (33b), the interchange of the + and - in a triplet pair does not give a minus sign. We expect the Néel amplitudes in the first excited state to be large (cf. fig. 3). Therefore we choose the signs so that in each triplet covering Néel 1 is given the coefficient +1 . As an example we consider the triplet state $|1\rangle$ of fig. $4 \mathrm{~b}$, in which the pair of spins 1 and 2 is in the triplet state. $|1\rangle$ can be written as

$$
|1\rangle=c\{|+-+-\rangle+|-++-\rangle-|+--+\rangle-|-+-+\rangle\} .
$$

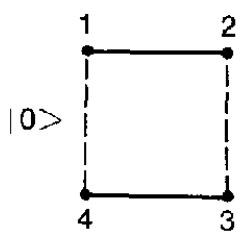

a)

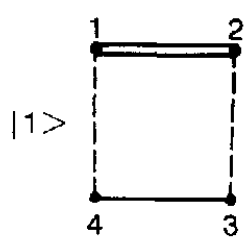

b)

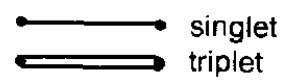

Fig. 4. a) Singlet covering, b) triplet covering. 
The first excited state of the ring of 4 spins can be obtained by simply adding the four possible triplet coverings, cf. fig. 5. A straightforward calculation gives

$$
\begin{aligned}
\left|\Psi_{1}\right\rangle= & |1\rangle+|2\rangle+|3\rangle+|4\rangle \\
= & c\{|+-+-\rangle+|-++-\rangle-|+--+\rangle-|-+-+\rangle\} \\
& +c\{|+-+-\rangle+|++--\rangle-|--++\rangle-|-+-+\rangle\} \\
& +c\{|+-+-\rangle+|+--+\rangle-|-++-\rangle-|-+-+\rangle\} \\
& +c\{|+-+-\rangle+|--++\rangle-|++--\rangle-|-+-+\rangle\} \\
= & 4 c\{|+-+-\rangle-|-+-+\rangle\},
\end{aligned}
$$

which is the exact first excited state (cf. (34)).

In general we approximate the first excited state by the following linear combination of triplet coverings:

$$
\left|\Psi_{1}\right\rangle=\sum_{n} \sum_{k}^{*} c_{n, k}|n, k\rangle .
$$

In (39) the first summation runs over all dimer (singlet) coverings $|n\rangle$, while for each $n$ the second one runs over all possible ways to replace one singlet pair in $|n\rangle$ by a triplet pair. We can also write $\left|\Psi_{1}\right\rangle$ as a linear combination of Ising configurations,

$$
\left|\Psi_{1}\right\rangle=\sum_{i} \varepsilon_{i}\left(\sum_{n} \sum_{k}^{*} c_{n, k} \xi_{i, n} \sigma_{i, k}\right)\left|\varphi_{i}\right\rangle,
$$

in which, besides the "indicator" $\xi_{i, n}$ (which takes the value $1(0)$ if $\left|\varphi_{i}\right\rangle$ can(not) be covered by the covering $|n, k\rangle)$, an extra sign $\sigma_{i, k}$, which depends on the "orientation" of the triplet pair, is added to the Marshall sign $\varepsilon_{i}=(-)^{p_{i}}$ of the coefficient for $\left|\varphi_{i}\right\rangle$. We define:

$-\sigma_{i, k}=+1$ for Ising configurations $i$, in which the + spin in the triplet pair in the triplet covering $k$ is situated on the $\alpha$ sublattice.

$-\sigma_{i, k}=-1$ for Ising configurations with the + spin situated on $\beta$.

Now we notice that for Néel 1 (2) we find $\sigma_{i, k}=+1(-1)$ for each $k$.

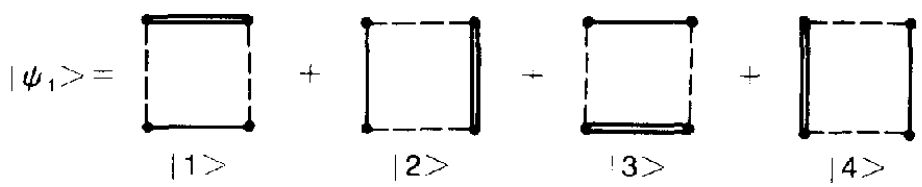

Fig. 5. Construction of the first excited state for a system of four spins. 
Furthermore it follows immediately that for each $k$ and for each Ising configuration $i$ and its time reversed $j$, we must have: $\sigma_{i, k}=-\sigma_{j, k}$, so it is clear that $\left|\Psi_{1}\right\rangle$ has the opposite TR symmetry, compared to $\left|\Psi_{0}\right\rangle$.

For Ising configurations "close" to Néel 1, i.e. with many + spins on the $\alpha$ sublattice, the number of triplet coverings $k$ with a "well-orientated" triplet dimer $\left(\sigma_{i, k}=+1\right)$ will exceed the number of "wrongly orientated" triplet coverings. In the land/water structure of $\left|\Psi_{1}\right\rangle$ we will therefore find a land area around Néel 1 and its time-reversed water area, including Néel 2. Some small deviations from this general pattern, e.g. some small areas, in the middle of the configuration network, may occur. These features are the same as those predicted in the sections 4 and 5 for the first excited state, so we have a first indication that our approximation may be a realistic one. Another check can be made by calculating the expectation value for the energy,

$$
E_{1}=\left\langle\Psi_{1}|\mathbf{H}| \Psi_{1}\right\rangle /\left\langle\Psi_{1} \mid \Psi_{1}\right\rangle .
$$

The accuracy of the method is expected to be dependent of the dimension $d$, as was the case for the approximation method for the ground state $^{5}$ ). We give some examples:

$d=\infty$ : In the spherical limit our method is exact.

$d=1$ : For an infinite one-dimensional lattice we get an energy -0.375 per spin (the same as the approximated ground-state energy), while it should have Hulthen's value: $\frac{1}{4}-\ln 2 \approx-0.443^{9}$ ). Clearly the number of coverings is too small to give a reliable approximation, as was the case for the ground state.

$d=2$ : We are not able to perform calculations for large two-dimensional lattices. Therefore we use the $4 \times 4$ square, with periodic boundary conditions, to investigate the working of the approximation in two dimensions. For an example of a possible triplet covering of this system, see fig. 6 .

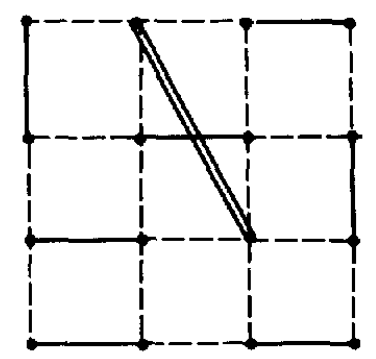

Fig. 6. A triplet covering for the $4 \times 4$ square. 
We investigated several cases: All triplet coverings with the triplet situated on a neighbour pair are given the weight $c_{1}$, while coverings with the triplet spins separated by a distance $\sqrt{5}$ (the knight's move) are given the weight $c_{2}$ (the singlet pairs always form neighbour pairs only). In table III we have given the results of these calculations. The minimum in the energy occurred for

$$
\left(c_{2} / c_{1}\right)_{\min }=0.31
$$

In table III we have also given the results for the approximated ground state, with weights $\tilde{c}_{1}$ (only neighbour dimers) and $\tilde{c}_{2}$ (one singlet pair at the knight's-move distance). For the ground state we also calculated the ratio $\tilde{c}_{2} / \tilde{c}_{1}$ for which the energy takes its minimum value. We found ${ }^{5}$ )

$$
\left(\tilde{c}_{2} / \tilde{c}_{1}\right)_{\min }=1.41
$$

From table III we may conclude that indeed the energy of the first excited state is somewhat higher than the ground-state energy (as it should be). Comparing the two best approximations, we find a difference in the order of $6 \%$.

One other thing can be seen from table III. We compare two approximations: the one with the triplet pair situated on neighbouring sites $\left(c_{1} \neq 0\right.$, $\left.c_{2}=0\right)$, and the one with this pair at a knight's move distance $\left(c_{1}=0, c_{2} \neq 0\right)$. One may expect the triplet spins to repulse each other, but this seems not to be the case, as can be concluded from the resp. energies. This, combined with the small value of the quotient (42), may be an indication that the triplet is bounded, which may be explained by the effect of resonance, while triplet coverings with a "knight's-move triplet" have not much overlap with other coverings.

$d=3$ : We also considered the $2 \times 2 \times 2$ cube. With the two types of coverings (total $12+12$ coverings), drawn in fig. 7 , we are able to construct the exact first excited state, so only coverings with the triplet situated on a neighbour pair of

TABLE III

Results for the $4 \times 4$ square with periodic boundary conditions.

\begin{tabular}{lclll}
\hline \multicolumn{2}{c}{ Ground state } & & \multicolumn{2}{c}{ First excitcd state } \\
\cline { 5 - 6 } Approximation & $\varepsilon$ & & Approximation & $\varepsilon$ \\
\hline$\tilde{c}_{1}=\tilde{c}_{2}$ & -0.6964 & & $c_{1}=c_{2}$ & -0.6544 \\
$\tilde{c}_{1} \neq 0, \quad \tilde{c}_{2}=0$ & -0.6686 & $c_{1} \neq 0, \quad c_{2}=0$ & -0.6556 \\
$\tilde{c}_{1}=0, \quad \tilde{c}_{2} \neq 0$ & -0.6958 & $c_{1}=0, \quad c_{2} \neq 0$ & -0.6231 \\
$\left(\tilde{c}_{1}, \tilde{c}_{2}\right)_{\text {min }}$ & -0.6965 & & $\left(c_{1}, c_{2}\right)_{\min }$ & -0.6569 \\
\hline
\end{tabular}



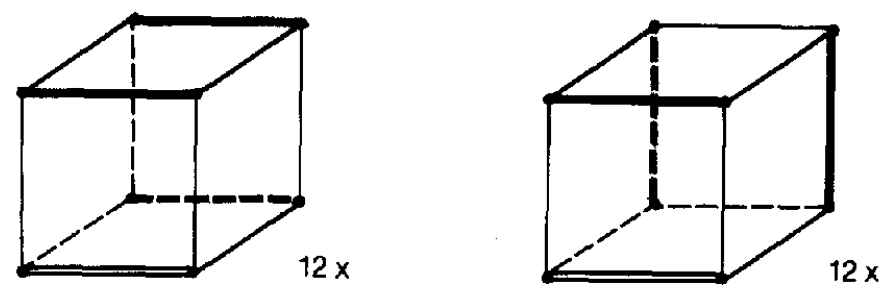

Fig. 7. The two types of coverings for the $2 \times 2 \times 2$ cube.

spins are needed. This was not the case for the ground state, where we got a difference of $2 \%$ between the exact and the approximated value.

The exact energy per spin in the ground state and first excited state are given by ${ }^{10}$ )

$$
\varepsilon_{0}=-0.603, \quad \varepsilon_{1}=-0.500
$$

The fact that we do not need to consider other types of coverings may be another indication that the spins, participating in the triplet, prefer to be close together.

\section{Conclusions, questions}

In the first section we have shown that there is a strong relation between Heisenberg systems and classical systems of coupled harmonic oscillators. The (eigen)states of the Heisenberg system can be characterized by a so-called land/water structure, defined on the corresponding configuration network, consisting of Ising configurations. These structures have features, which can be made plausible by comparing with the more familiar features of the equivalent harmonic-oscillator systems. The first excited state seems always to be a triplet state, but we could not find a rigorous proof for this statement. In the last sections we introduced an approximation method to describe elementary excitations. This method, which may be seen as a generalization of a method to describe the ground state, introduced in a previous work, is based on dimer coverings. The method, which is expected to be more accurate for higher dimensions, may explain the absence of an energy gap in the spectrum of spin- $\frac{1}{2}$ antiferromagnetic Heisenberg systems. If the amplitudes, corresponding to Ising configurations in the neighbourhood of the node (that occurs in the structure of the first excited state), are small enough, we may expect the absence of such a gap. Furthermore there are indications that the triplet pairs 
in the coverings are bounded, i.e. spins, joined in the triplet, prefer to be close together. Calculations for large systems will be necessary to decide whether these conjectures are true or not. Higher excited states may be described by introducing more triplet pairs, quintuplet squares, etc. In these cases interactions between such triplet pairs, squares etc., should be taken into account.

\section{Appendix}

In table A.I we have listed the 20 Ising configurations corresponding to systems with 6 spins. Notice that the time-reversed configuration, belonging to $\left|\varphi_{i}\right\rangle$, is $\left|\varphi_{21 \cdots i}\right\rangle$.

TABI.E A.I

The 20 Ising configurations corresponding to a Heisenberg system with 6 spins.

\begin{tabular}{|c|c|}
\hline $\begin{array}{l}\left|\varphi_{1}\right\rangle=|+++---\rangle \\
\left\{\varphi_{2}\right\rangle=|++-+--\rangle \\
\left|\varphi_{3}\right\rangle=|++--+-\rangle \\
\left|\psi_{4}\right\rangle=|++---+\rangle \\
\left|\psi_{5}\right\rangle=|+-++--\rangle \\
\left|\varphi_{6}\right\rangle=|+-+-+-\rangle \\
\left|\varphi_{7}\right\rangle=|+-+--+\rangle \\
\left|\varphi_{8}\right\rangle=|+--++\rangle \\
\left\{\varphi_{1}\right\rangle=|+--+-+\rangle \\
\left|\varphi_{10}\right\rangle=|+--++\rangle\end{array}$ & $\begin{array}{l}\left|\varphi_{20}\right\rangle=|---+++\rangle \\
\left\{\varphi_{19}\right\rangle=|--+-++\rangle \\
\left|\varphi_{18}\right\rangle=|--++-+\rangle \\
\left|\psi_{19}\right\rangle=|--+++\rangle \\
\left|\psi_{16}\right\rangle=|-+--++\rangle \\
\left|\varphi_{13}\right\rangle=|-+-+-+\rangle \\
\left|\varphi_{14}\right\rangle=|-+-++-\rangle \\
\left|\varphi_{13}\right\rangle=|-++-++\rangle \\
\left\{\psi_{12}\right\rangle=|-++-+-\rangle \\
\left|\varphi_{11}\right\rangle=|-+++--\rangle\end{array}$ \\
\hline
\end{tabular}

TABLE A.II

Land iwater structure of the three Heisenberg systems with 6 spins.

\begin{tabular}{|c|c|c|c|c|c|c|c|c|c|}
\hline \multicolumn{2}{|c|}{ Spherical } & & & \multicolumn{3}{|c|}{ Ring } & & \multicolumn{2}{|c|}{ Chain } \\
\hline Land & Water & & & Land & Water & & & Land & Water \\
\hline$\left|\varphi_{1}\right\rangle$ & $\left|\varphi_{20}\right\rangle$ & * & * & $\left|\varphi_{20}\right\rangle$ & $\left|\varphi_{1}\right\rangle$ & $*$ & * & $\left|\varphi_{1}\right\rangle$ & $\left|\varphi_{20}\right\rangle$ \\
\hline$\left|\varphi_{19}\right\rangle$ & $\left|\varphi_{2}\right\rangle$ & & & $\left|\varphi_{19}\right\rangle$ & $\left|\varphi_{2}\right\rangle$ & 4 & * & $\left|\varphi_{2}\right\rangle$ & $\left|\varphi_{19}\right\rangle$ \\
\hline$\left|\varphi_{3}\right\rangle$ & $\left|\varphi_{18}\right\rangle$ & & & $\left|\varphi_{3}\right\rangle$ & $\left|\varphi_{18}\right\rangle$ & & & $\left|\varphi_{3}\right\rangle$ & $\left|\varphi_{1:}\right\rangle$ \\
\hline$\left|\varphi_{17}\right\rangle$ & $\left|\varphi_{4}\right\rangle$ & $*$ & * & $\left|\varphi_{4}\right\rangle$ & $\left|\varphi_{17}\right\rangle$ & & & $\left|\varphi_{4}\right\rangle$ & $\left|\varphi_{17}\right\rangle$ \\
\hline$\left|\varphi_{5}\right\rangle$ & $\left|\varphi_{16}\right\rangle$ & & & $\left|\varphi_{5}\right\rangle$ & $\left|\varphi_{16}\right\rangle$ & & & $\left|\varphi_{5}\right\rangle$ & $\left|\varphi_{10}\right\rangle$ \\
\hline$\left|\varphi_{6}\right\rangle$ & $\left|\varphi_{15}\right\rangle$ & & & $\left|\varphi_{6}\right\rangle$ & $\left|\varphi_{15}\right\rangle$ & & & $\left|\varphi_{6}\right\rangle$ & $\left|\varphi_{15}\right\rangle$ \\
\hline$\left|\varphi_{7}\right\rangle$ & $\left|\varphi_{14}\right\rangle$ & & & $\left|\varphi_{7}\right\rangle$ & $\left|\varphi_{14}\right\rangle$ & & & $|\varphi\rangle$, & $\left|\varphi_{14}\right\rangle$ \\
\hline$\left|\varphi_{8}\right\rangle$ & $\left|\varphi_{13}\right\rangle$ & & & $\left|\varphi_{R}\right\rangle$ & $\left|\varphi_{13}\right\rangle$ & & & $\left|\varphi_{y}\right\rangle$ & $\left|\varphi_{13}\right\rangle$ \\
\hline$\left|\varphi_{12}\right\rangle$ & $\left|\varphi_{9}\right\rangle$ & & & $\left|\varphi_{12}\right\rangle$ & $\left|\varphi_{y}\right\rangle$ & & & $\left|\varphi_{12}\right\rangle$ & $\left|\varphi_{y}\right\rangle$ \\
\hline$\left|\varphi_{10}\right\rangle$ & $\left|\varphi_{11}\right\rangle$ & * & * & $\left|\varphi_{11}\right\rangle$ & $\left|\varphi_{10}\right\rangle$ & & & $\left|\varphi_{11}\right\rangle$ & $\left|\varphi_{10}\right\rangle$ \\
\hline
\end{tabular}



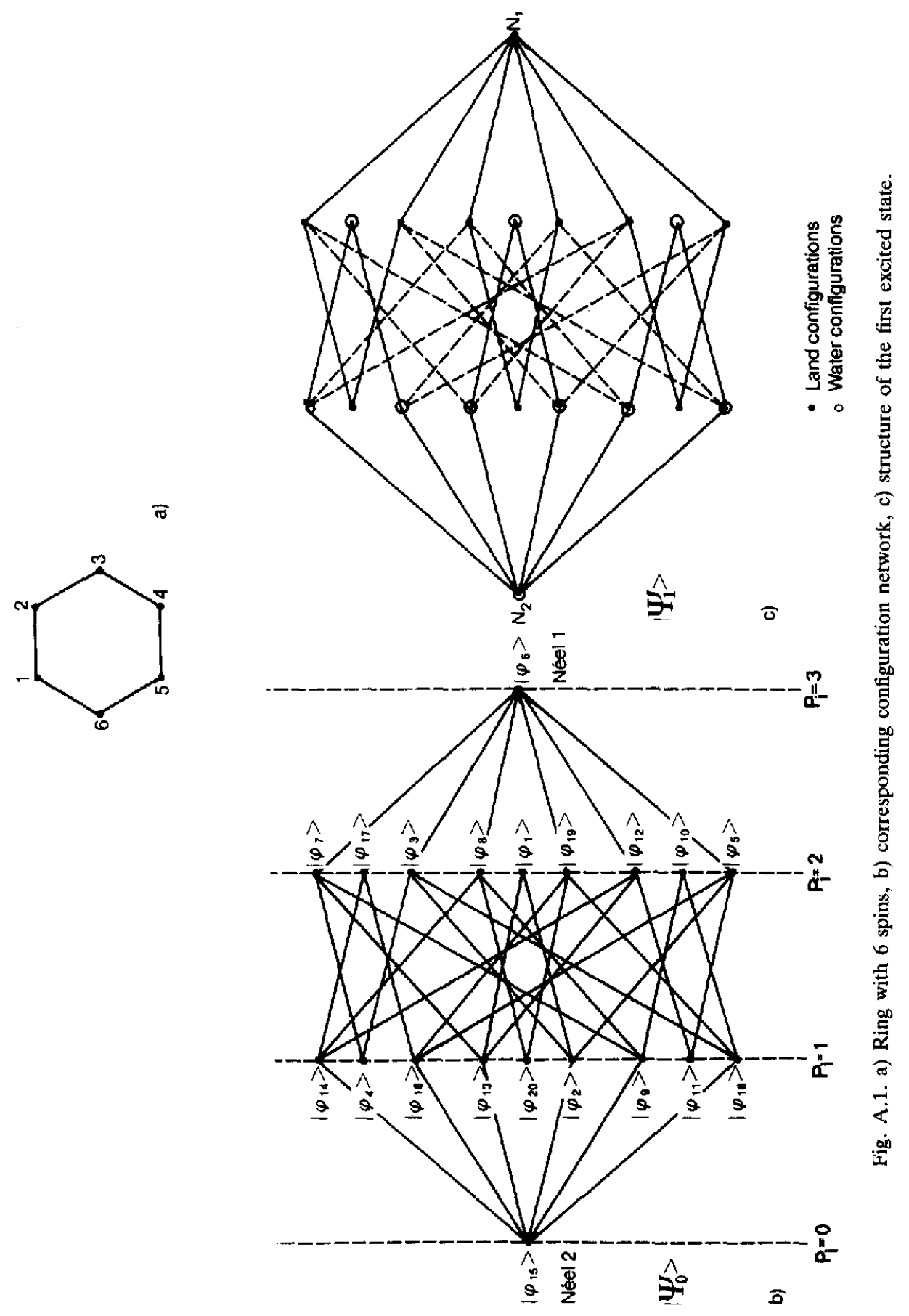
In fig. A1.b we have drawn the configuration network, corresponding to the Heisenberg system situated on a ring with 6 spins (cf. fig. A.1a). In fig. A.1c we have given the land/water structure, corresponding to the first excited state of this system. In fig. A.1b,c all Ising configurations with the same $p_{i}$, i.e. with the same sublattice magnetization, are situated in the same column.

In table A.II we have given the land/water structure, corresponding to the first excited state of the three Heisenberg systems with 6 spins (spherical latticc, ring with 6 spins, chain with 6 spins). The total spin in all three cases has the value $S=1$.

In table A.II the differences between the spherical model and the model on the ring are denoted with an *. The involved lsing configurations are all equivalent on grounds of translational invariance. One can cxplain for these configurations to belong to the reversed area in the two systems: In the configuration network, corresponding to the spherical model, there exists a direct connection between e.g. $\left|\varphi_{1}\right\rangle$ and the first Néel configuration $\left|\varphi_{6}\right\rangle$. These configurations can be obtained from each other by interchanging the spins 2 and 5 . In the ring, the diagonal bond $(2,5)$ docs not exist any more. This implies for the configuration network that $\left|\varphi_{1}\right\rangle$ is no longer close to Néel 1. The same remarks can be made for the configurations marked by a ". These configurations are shifted to the inversed area, because of deleting the bond $(1,6)$ from the ring-lattice. As an example we consider the (connected) configurations $\left|\varphi_{1}\right\rangle$ and $\left|\varphi_{2}\right\rangle$. While there is no connection between $\left|\varphi_{1}\right\rangle$ and $\left|\varphi_{13}\right\rangle$, and neither between $\left|\varphi_{2}\right\rangle$ and $\left|\varphi_{15}\right\rangle$, in the network corresponding to the chain, $\left|\varphi_{1}\right\rangle$ and $\left|\varphi_{2}\right\rangle$ do not belong to the water area any more.

\section{References}

1) H. Bethe, Z. Physik 71 (1931) 205.

2) N.D. Mermin and H. Wagner, Phys. Rev. Lett. 17 (1966) 1133.

3) W. Marshall, Proc. Roy. Soc. (London) A 232 (1955) 48.

4) J. des Cloizeaux and J.J. Pearson, Phys. Rev. 128 (1962) 2131.

5) P.L. Iske and W.J. Caspers, Physica 142A (1987) 360.

6) G. Kirchhoff, Ann. Phys. Chem. 72 (1847) 497.

7) E. Lieb and D. Mattis, J. Math. Phys. 3 (1962) 749.

8) A.R. Edmonds, Angular Momentum in Quantum Mechanics (Princeton Univ. Press, Princeton, 1957), sees. 2.8 and 3.5 .

9) L. Hulthen, Arkiv Mat. Astron. Fysik 26A (1938) 11.

10) W.J. van den Broek, private communication, University of Twente, Enschede (1982). 